\title{
Changes in hand surgery in Canada over the past 40 years: Some personal observations
}

\author{
Arnis Freiberg MD FRCSC FACS
}

$\mathrm{H}$ nd surgery has changed over the years - not only in Canada, but also worldwide. Some aspects have changed significantly, while others have stayed the same. Although most of the changes have had a very positive influence on treatment outcomes, there have been some aspects of these changes that have had a negative effect and are of some concern for the future.

\section{HAND SURGERY IN CANADA VERSUS THE UNITED STATES}

Since the introduction of universal health care in Canada approximately 30 years ago, every patient with a hand injury has received the same care, regardless of their financial status or their place of employment. Although future changes to the health care system in the United States (US) may occur during the current administration, it does not appear that every patient with a hand injury will be able to receive the same care without incurring a cost, which could be prohibitive. In the US, a patient with a hand injury can receive treatment in a private outof-hospital unit; in Canada, this is neither practical nor desirable. Hand surgeons in the US usually have to deal with third-party representatives who often are the decision makers regarding whether the proposed surgery will be covered by their company. Fortunately, this is not the case in Canada.

\section{HAND SURGERY PROGRAMS}

Approximately 40 years ago, in Toronto, Ontario - an area that I am most familiar with - most of the hand surgery procedures were performed by plastic surgeons, in both teaching and community hospitals. Wrist surgeries were performed by local orthopedic surgeons because most of the plastic surgeons at that time were not well trained in this area of the body.

I believe that some very significant and positive changes have occurred since that time. There has been a very healthy and beneficial cross-fertilization between the plastic and orthopedic surgery training programs and their surgeons. The plastic surgery residents, at least at the University of Toronto, are now well trained in wrist surgery, and the orthopedic residents are now exposed to hand surgery early in their residency program.

We now also have specialized hand and upper extremity units that have both plastic and orthopedic surgeons who provide not only expert tertiary care, but also train residents, medical students and fellows from all over the world. In these units, microsurgical training is also provided to residents from both disciplines.

\section{THE EFFECTS ON HAND SURGERY DUE TO SUBSPECIALIZATION IN PLASTIC AND ORTHOPEDIC SURGERY}

\section{Plastic surgery}

Although cosmetic surgery has been performed by plastic surgeons for a long time, over the past 10 to 15 years, more community surgeons are either confining their practices to cosmetic surgery in free-standing private units or spending a considerable percentage of their time performing cosmetic surgeries. This, I believe, has produced a negative influence on the field of hand surgery, especially within the community. Previous referrals to the University of Toronto's Hand Surgery Program mainly consisted of complex problems, at least in my practice; however, over the past five to 10 years, referrals have been for simple problems such as trigger fingers! It is not uncommon for patients to be surprised that plastic surgeons treat hand problems because most people believe that plastic surgery implies cosmetic surgery.

\section{Orthopedic surgery}

Subspecialization in orthopedic surgery has also had a negative influence on hand surgery. Hip and knee replacement surgeries pay better than hand and wrist surgeries! As a result, orthopedic hand surgeons in our program are seeing more simple problems such as undisplaced wrist fractures.

\section{HAND THERAPISTS AND HAND REHABILITATION}

Forty years ago at the University of Toronto, there were two separate faculties: physiotherapy and occupational therapy. Today, the two faculties have merged. Hand therapists in our institution offer both therapy and make orthotics; they are simply referred to as 'hand therapists'. A few years previously, there were only regional hand therapy interest groups; we now have a Canadian national organization that holds annual meetings. Hand therapists in the US have their annual meetings at the same time as the Annual Hand Society and Hand Association meetings. In April 2010, for the first time, a very successful combined meeting was held in Montreal, Quebec, between the Canadian Society of Hand Therapy and Manus Canada. For many years, there was a generally accepted belief that good outcomes in hand surgery were due to $70 \%$ hand therapy and 30\% surgery. This is one concept that has not changed.

\section{MICROSURGERY AND REPLANTATION}

There is no doubt that the development of microsurgery is one of the most significant milestones in hand surgery. It has not only enabled us to repair small vessels, but also significantly improved the results in nerve repairs. It has also allowed us to reattach severed parts such as fingers and hands. However, there is a Latvian saying that "freshly baked bread also comes with a crust". Although the technical aspects of replantation approaches perfection, there are also some downsides. It has been said that "if you have a hammer, the whole world looks like a nail". Some surgeons have a tendency to replant everything, especially single, nondominant digits. The result is often a poorly functioning digit that sometimes affects the function of the rest of the hand. Even today, we have problems achieving consistently good results from tendon, bone and nerve repairs alone! When we combine all of these in a single replanted digit, we often end up with a relatively poor result.

\section{HAND TRANSPLANTATION}

There have been several reports from around the world that have described variable results with regard to hand transplantation and, thus, the topic still remains controversial. There is no problem with the technical skills required in transplantation; however, the prolonged 
commitment to very powerful antirejection drugs involves some difficult decisions for both the patient and the surgeon. There is no doubt that these drugs and their side effects are justifiable in life-threatening organ transplants, but are they justifiable in a hand transplant? Further developments in this field are needed from the transplant specialists.

\section{PERIPHERAL NERVES}

The technical aspects of fascicular repairs have significantly improved the results of nerve repairs over the past 40 years. Intraoperative nerve stimulation has enabled us to connect the right fascicles both in nerve repairs and nerve transfers. Recognition of the importance of early nerve gliding following nerve repairs and nerve decompression has also improved the functional results. Recognition of the 'double crush' syndrome has resulted in a search for other more proximal causes for tingling in the fingers rather than labelling it as 'carpal tunnel syndrome' (CTS). The greatest and most important discovery over the past few years, however, has been the ability to perform nerve transfers - a term that was previously only used for tendons.

\section{JOINT AND JOINT REPLACEMENT}

The Swanson silicone spacers were the 'gold standard' for many years, especially for finger joints, and are still used today. Because of breakage, especially in high-demand joints, such as the metacarpophalangeal joint of the index finger in the rheumatoid patient, there is still ongoing research for other materials such as metal or metal derivatives. The main problem is the high cost and the lack of long-term follow-up studies. The Swanson spacers in the carpus were abandoned because of serious silicone synovitis. For a while, there was some enthusiasm for autologous tissue joint replacements such as perichondral grafts. However, for many reasons, these grafts did not fulfill the initial expectations. The search for the ideal total wrist prosthesis, which began approximately 30 years ago, is still ongoing. For as long as I can remember, each year at the annual hand society meetings, a new prosthesis is presented by the Mayo hand group, simply meaning that none of the models of the previous years were satisfactory!

\section{TENDONS AND TENDON TRANSFERS}

It is interesting to note that there have not been any significant changes over the past 40 years, either in extensor or flexor tendon repairs or tendon transfers. Although there has been some improvement in suture materials and minor changes in postoperative hand therapy, none of these changes have significantly affected the outcomes; adhesion and rupture problems are still present. The Hunter tendon rod implant is still popular, and the basic rules and requirements in tendon transfer surgery, which were proposed many years ago, have withstood the test of time.

\section{DUPUYTREN'S DISEASE AND CONTRACTURES}

The etiology of Dupuytren's disease was found to be genetically based following the discovery and identification of a specific genetic locus. The relationship between heavy alcohol intake and Dupuytren's disease has been disputed since the discovery of the above condition. The northern Europeans have been known to be heavy drinkers! Despite extensive research, no effective nonsurgical treatment has been found. Limited palmar fasciectomy still remains the 'gold standard' in surgical management. As a resident, I had the chance to work with a staffman who believed in total palmar fasciectomy. Lesson learned: don't do it!

\section{CTS}

As a resident, I only saw advanced and neglected cases. For the past decade, CTS has been seriously overdiagnosed. Almost every referral with numbness or tingling is labelled as having CTS. The role of nerve conduction studies and electromyography in diagnosis has become quite controversial. The advent of minimally invasive surgery in other disciplines prompted a wave of enthusiasm for endoscopic carpal tunnel release. The arguments related to 'open versus closed' carpal tunnel release surgery still exist; however, the enthusiasm for 'closed' carpal tunnel release surgery has slowed considerably. The advent of the diagnosis of 'double crush' syndrome has made us aware of the importance of examining the neck for coexisting thoracic outlet syndrome, cervical radiculopathy and poor posture in cases in which the referring diagnosis is CTS. Repetitive use as a cause for CTS is still controversial. Why does the low-demand hand in an elderly woman develop CTS? The importance of early postoperative movement to obtain nerve gliding is gaining in popularity.

\section{AESTHETIC PROSTHESES}

There has been significant improvement in the quality and appearance of aesthetic prostheses. However, there are some significant problems aside from cost - that are prohibitive for noncompensible individuals. Because of the lack of sensation at the finger tips, these prostheses are used for aesthetic purposes only. Finger prostheses also pose a danger when operating moving machinery.

\section{SYNDROMES: OLD AND NEW}

The old term 'reflex sympathetic dystrophy' has undergone some significant and beneficial changes. The bone scan as a diagnostic tool is no longer accepted. The term itself has been replaced by chronic regional pain syndrome type 1 , which has three subtypes, replacing the minor and major causalgia. Benign hypermobile joint syndrome has provided us with an explanation for the symptoms of pain and joint instability in the young patient. We now recognize that the psychiatric diagnosis of 'somatization disorder' can also be confined to the upper extremity, explaining some of the atypical pain observed in patients with no apparent anatomical basis.

\section{THE FUTURE OF HAND SURGERY: WHAT CAN WE EXPECT OVER THE NEXT 40 YEARS?}

\section{Hand transplantation}

Further improvements will be in the hands of immunologists and individuals dealing with ethics.

\section{Musculoskeletal imaging}

The $\mathrm{x}$-ray will remain a valuable diagnostic tool; however, the computed tomography scan has now evolved from a two-dimensional to a three-dimensional model. The role of magnetic resonance imaging in diagnosing soft tissue problems in the hand and wrist is gaining in popularity; however, we must be careful in drawing diagnostic and clinical conclusions because we are now seeing details that may well be within normal limits. The use of ultrasound - a quick and inexpensive diagnostic tool - will still play a significant role in hand practice. With these newer developments, we will need the assistance of musculoskeletal imaging specialists because most of these studies will be operator dependant.

Using high-tech magnetic resonance imaging scans of the brain, and functional and dynamic studies, we can better understand the brain in relation to the function of the hand. Further research is not only exciting, but may be helpful in treatment. A study by Taylor et al (1) illustrates the exciting future developments in hand surgery.

The application of stem cell research to the hand may, in the future, enable us to regenerate missing parts in the hand.

\section{REFERENCE}

1. Taylor KS, Anastakis DJ, Davis KD. Cutting your nerve changes your brain. Brain 2009;132:3122-33. 